\title{
Vanishing Point Detection in the Hough Transform Space
}

\author{
Andrea Matessi and Luca Lombardi \\ Dipartimento di Informatica e Sistemistica, University of Pavia, \\ Via Ferrata 1, I-27100 Pavia (Italy). \\ e-mail: matessi@stigma.it, llombardi@dis.unipv.it
}

\begin{abstract}
Depth estimation from monocular images can be retrieved from the perspective distortion. One major effect of this distortion is that a set of parallel lines in the real world converges into a single point in the image plane. The estimation of the co-ordinates of the vanishing point can be retrieved directly on the Hough Transformation space or polar plane. In fact the vanishing point in the image plane is mapped in the polar plane into a sine curve that can be estimated with a simple linear system.
\end{abstract}

\section{Introduction}

In monocular images depth estimation, if no particular prior knowledge of the scene is given, can be retrieved in many real images by the perspective distortion. One major effect is that a set of parallel lines in the three dimensional space in the image space converges to a single point called the vanishing point. This point in the image plane gives important information on the distance of the objects in the scene and of the three--dimensional structure. In Sedgwick [2] some features of the vanishing point are given. Since this point is the projection of a point at the infinite with respect to the point of observation, a finite motion of the point of observation produces a movement of the vanishing point in the image that is negligible. This is called invariance to motion. This feature must be taken into account when considering sequential images of a moving point of observation.

In this work an analytical method to determine the vanishing point in the Hough Parameters Space is presented. This method is computationally equivalent to others but has the advantage that in the Hough Plane the vanishing point has a precise shape that can be searched optimally. The algorithm to detect the vanishing point was also implemented and the results from real images are shown. This same algorithm can be used to detect the focus of expansion in moving scenes to determine the speed of objects in the three dimensional space.

The next section provides a brief background on the problem and a few previous solutions. Section 3 outlines the proposed approach. Section 4 shows our preliminary results. Finally, conclusions and directions for the future work are stated. 


\section{Perspective Projection and Notations}

Let us analyse the perspective projection and the transformation of a point of the real world into the image. The scene or the camera co-ordinates are set to define the Cartesian system XYZ where the Z-axis is the camera optical axis. Let the point $(0,0,-f)$ be the viewpoint or the centre of the lens of the camera. Thus the image plane $(x, y)$ coincides with the XY-plane. The parameter $f$ refers to the focal length.

In perspective projection a point in the real world $(\mathrm{X}, \mathrm{Y}, \mathrm{Z})$ is mapped onto the intersection $(x, y)$ of the XY-plane with the straight line that passes through the $(\mathrm{X}, \mathrm{Y}, \mathrm{Z})$ point and the viewpoint. From Fig. 1 and from the similitude of triangles it is not difficult to show that the transformation $(\mathrm{X}, \mathrm{Y}, \mathrm{Z})$ to $(x, y)$ is given by:

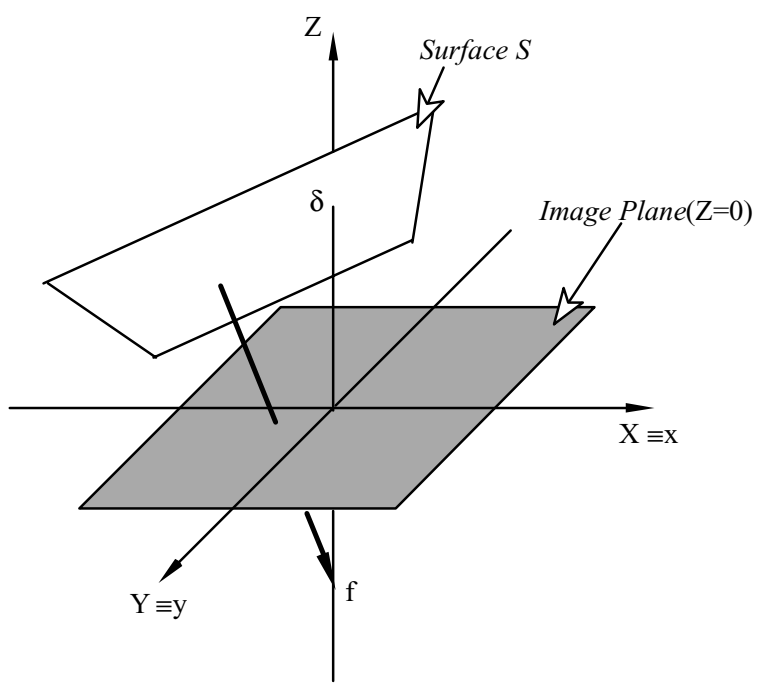

Fig. 1. The model of the perspective projection.

$$
x=\frac{f X}{f+Z}, \quad y=\frac{f Y}{f+Z}
$$

Analysing the transformations in (1) and by experience it is clear that a set of parallel lines in the real world converges to a single point in the image plane. This point corresponds to the projection of the line in the set that passes through the point of observation. This point is called the vanishing point. In other words to the orientation of a set of parallel lines there corresponds a single vanishing point and, conversely, to a vanishing point there corresponds an orientation.

The detection of the co-ordinates of a vanishing point in the scene can provide relevant information on the depth of a single image, on the distance of objects in a plane, or also on the height of objects in the scene.

It is clear that to determine the vanishing point, at least two straight lines on the scene are necessary. 


\subsection{Hough Transformation}

In this section we will describe how a straight line is detected in an image plane using the Hough Transform [6, 7].

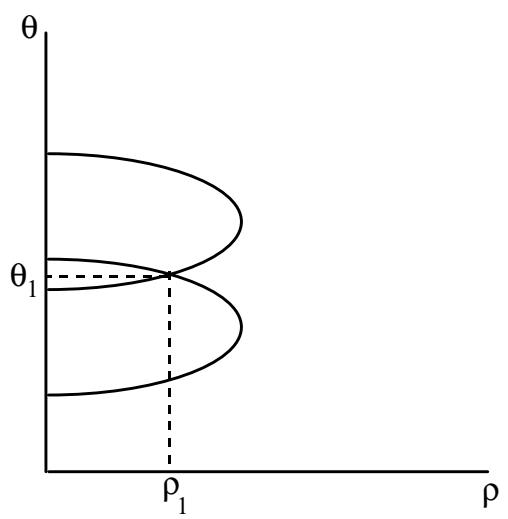

Fig. 2: Straight line detection with HT: the intersection of two sinusoidal curves determines the parameters of a line.

Hough Transform (HT) for straight lines detection consists in mapping the image plane into a parameters plane. A straight line can be therefore described through a parametric representation. The two most common representations are the following:

$$
\rho=x \cos \theta+y \sin \theta, \quad y=\mathrm{m} x+\mathrm{q} .
$$

The first formula in many cases is considered to be more convenient for the purposes of the present work, the two parameters $\rho$ and $\theta$ are defined in limited ranges. In fact $\rho$ is bound by the dimensions of the image, while $\theta$ takes values in the $[0,2 \pi[$ range.

Let us analyse the detection process of a straight line in the image plane through the parameter space that in the case of the first formula in (2) is called the polar plane. To each point $\left(x_{\mathrm{i}}, y_{\mathrm{i}}\right)$ we can associate a sine curve $\rho\left(\theta, x_{\mathrm{i}}, y_{\mathrm{i}}\right)$ in the plane $(\rho$, $\theta)$. Two points in the image plane that belong to the same line determine a point in the polar plane $\left(\rho_{\mathrm{i}}, \theta_{\mathrm{i}}\right)$, given by the intersection of two sine curves. The straight line is therefore characterised by this point (Fig. 2).

This deterministic method is in real cases not useful because of noise in the image and because of the finite granularity of an image. In many cases, however, a rough probabilistic method is enough to determine a line with sufficient precision. A line then is determined by a threshold on the points in the polar plane that are mapped from the image plane. The threshold can be relative to the maximum number of points $\left(x_{\mathrm{i}}, y_{\mathrm{i}}\right)$ that determine a single couple $\left(\rho_{\mathrm{i}}, \theta_{\mathrm{i}}\right)$. 


\subsection{Previous Work on the Detection of the Vanishing Point}

Different algorithms have been implemented to determine the vanishing point in an image [7].

A first method implemented by Nakatani [1] uses the Hough Transformation to detect the straight lines in the polar plane as described above. The lines are then marked on a new image. The point where all the lines converge is the vanishing point. This is a deterministic method that is affected by many errors and in most cases there is not a single point where all the lines converge.

A second one is less deterministic. Here, the point that has the least distance forms all the lines in the image is considered to be the vanishing point. The minimum of the distance function is determined with a least square method.

What all these algorithms have in common is a double transformation. First the image is mapped in the polar plane with HT; then the polar plane is traced back to the image plane by means of some filtering.

In the new algorithm that is presented here, the vanishing point is detected directly on the polar plane. This has the advantage, as we will see later, that we search for a curve whose equation is clearly known and depends on the parameters that give us the position of the vanishing point. This fact helps us to do further approximations on the estimates. This algorithm is based on statistical assumptions that allows us to process noisy images. Further, acting directly on the polar plane, vanishing points that are outside the image do not need any special treatment. At present we are analysing how this algorithm must be modified so that multiple vanishing points can be recovered. Moreover, in the approach that detect the vanishing point from the original image, the choice of the hardness of filtering in the polar plane needed to detect straight lines has to be chosen in relation to the image. In the method that we present, filtering in the polar plane is not needed, thus the method is independent from the image.

\section{Vanishing Point Estimation in the Polar Plane}

A point given by the intersection of two straight lines in the image plane it is mapped into two points in the polar plane, each one of them representing one of the two straight lines in the image plane. These two points in the polar plane belong to the same arch of a sine curve. In fact, if we take the point $\left(x_{0}, y_{0}\right)$, centre of a set of intersecting straight lines, on the polar plane the following function is traced:

$$
\rho(\theta)=x_{0} \cos \theta+y_{0} \sin \theta=\mathrm{P}\left(x_{0}, y_{0}, \Phi\right) \sin \left(\theta+\Phi\left(x_{0}, y_{0}\right)\right)
$$

This is a sine curve with amplitude $\mathrm{P}=x_{0} \cos (\Phi)\left(1+y_{0}^{2} / x_{0}^{2}\right)$, and phase $\Phi=\arctan \left(y_{0} / x_{0}\right)$ (Fig. 3).

Thus the vanishing point is identified by the sine curve. In general in an image there are many straight lines that can not contribute to the vanishing point. Moreover there can be errors due mostly to the quantization of the image and to lines detection. Thus a deterministic approach to find the equation of the sine curve in not satisfac- 
tory. For this reason it is necessary to implement a statistical method. In this paper we adopted a least square method to find the pair of parameters $\left(x_{0}, y_{0}\right)$ that gives us the sine curve.

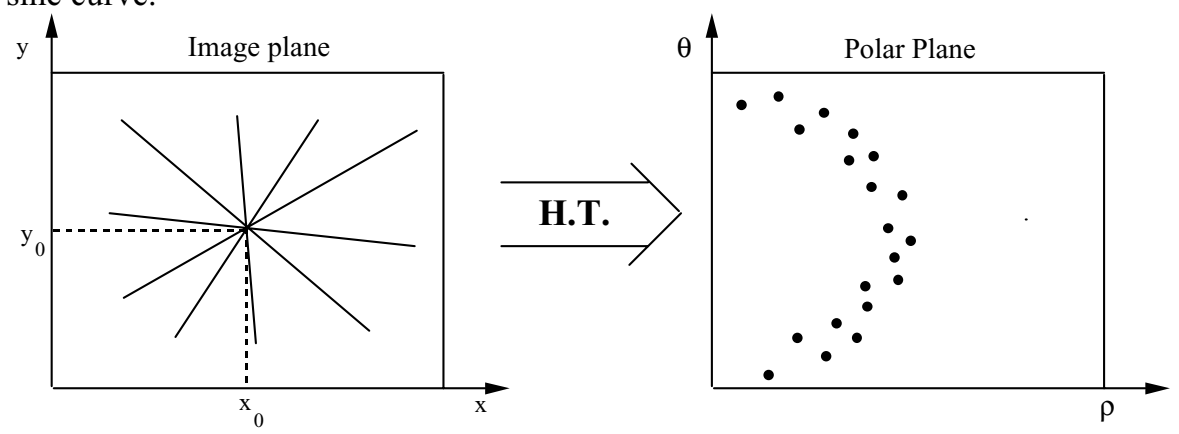

Fig. 3: Hough Transformation of a set of lines that intersect in a single point

The parameters that we have to estimate, $x_{0}$ and $y_{0}$, represent the co--ordinates of the vanishing point in the image. The estimation is made from a set of measures $\rho_{i}$ and $\theta_{\mathrm{i}}$ expected to lie on the curve defined by equation (3). The points with coordinates $\left(\rho_{i}, \theta_{i}\right)$ are weighted by $\mathrm{W}_{\mathrm{i}}$ given by the ratio $\mathrm{v}_{\mathrm{i}} / \mathrm{V}$, where $\mathrm{v}_{\mathrm{i}}$ is the number of times that the pair $\left(\rho_{\mathrm{i}}, \theta_{\mathrm{i}}\right)$ is observed as a mapping point of a line in the image, and $\mathrm{V}$ is the sum of all these. The least square minimisation is thus:

$$
\min _{x_{0}, y_{0}} \sum_{i=1}^{n} \mathrm{~W}_{i}\left(\rho_{i}-x_{0} \cos \theta_{i}-y_{0} \sin \theta_{i}\right)^{2}
$$

where $n$ is the total number of samples. If now we set $\cos \theta_{\mathrm{i}}=\mathrm{a}_{\mathrm{i}}$ and $\sin \theta_{\mathrm{i}}=\mathrm{b}_{\mathrm{i}}$ deriving with respect to $x_{0}$ and to $y_{0}$ and equating to zero, we get the following linear system:

$$
\left\{\begin{array}{l}
\sum \mathrm{W}_{\mathrm{i}} \mathrm{a}_{\mathrm{i}}\left(\rho_{\mathrm{i}}-\mathrm{a}_{\mathrm{i}} x_{0}-\mathrm{b}_{\mathrm{i}} y_{0}\right)=0 \\
\sum \mathrm{W}_{\mathrm{i}} \mathrm{b}_{\mathrm{i}}\left(\rho_{\mathrm{i}}-\mathrm{a}_{\mathrm{i}} x_{0}-\mathrm{b}_{\mathrm{i}} y_{0}\right)=0
\end{array}\right.
$$

Summing the left hand side of the two equations and making the following substitutions:

$$
\begin{gathered}
\mathrm{A}=\sum \mathrm{W}_{\mathrm{i}} \mathrm{a}_{\mathrm{i}}^{2} \quad \mathrm{~B}=\sum \mathrm{W}_{\mathrm{i}} \mathrm{b}_{\mathrm{i}}^{2} \quad \mathrm{C}=\sum \mathrm{W}_{\mathrm{i}} \mathrm{a}_{\mathrm{i}} \mathrm{b}_{\mathrm{i}} \\
\mathrm{D}=\sum \mathrm{W}_{\mathrm{i}} \mathrm{a}_{\mathrm{i}} \rho_{\mathrm{i}} \quad \mathrm{E}=\sum \mathrm{W}_{\mathrm{i}} \mathrm{b}_{\mathrm{i}} \rho_{\mathrm{i}}
\end{gathered}
$$

the following linear system is obtained:

$$
\left\{\begin{array}{l}
A \mathrm{x}_{0}+C \mathrm{y}_{0}=D \\
C \mathrm{x}_{0}+B \mathrm{y}_{0}=E
\end{array}\right.
$$

Thus the estimate is straightforward.

After the first estimate of the co-ordinates of the vanishing point is obtained, the previous process is repeated after elimination of the outliers. Outliers in the polar plane are identified in the following way. From the estimate of the vanishing point 
$\left(x_{0}, y_{0}\right)$ and for each $\theta_{\mathrm{i}}$ the estimated value of $\rho_{\mathrm{i}}, \bar{\rho}_{i}$, is calculated from (3); at this point the variance of the residuals $\varepsilon_{\mathrm{i}}=\rho_{\mathrm{i}}-\bar{\rho}_{i}$ is:

$$
\sigma^{2}=\sum_{i} W_{i} \varepsilon_{i}^{2} .
$$

Thus the outliers are all those points $\rho_{\mathrm{i}}$ of the polar plane that satisfy the following condition:

$$
\left|\rho_{i}-\bar{\rho}_{i}\right|>k \sigma
$$

where $k$ is generally taken between 2 and 3 (the number of outlies increases as $k$ decreases). Elimination of the outliers is done by setting to zero the points that are external to a band around the sine curve identified by the first estimate of the vanishing point.

Once the outliers are eliminated, a new estimate of the vanishing point is retrieved from, the result, obtaining the estimate $\left(x_{1}, y_{1}\right)$. The loop ends when:

$$
\left|\mathrm{x}_{i+1}-\mathrm{x}_{i}\right|+\left|\mathrm{y}_{i+1}-\mathrm{y}_{i}\right| \leq \varepsilon
$$

where $\varepsilon$ can be chosen depending on the precision of the estimate desired.

If the polar plane is dimensioned dynamically the case of a vanishing point that is much outside the image can be resolved without any particular problem. The extension to the case in which the vanishing points are more than one can instead be quite difficult unless the exact number is known.

\section{Results}

In this section some of the results obtained with this algorithm are shown. Most of the images are taken form Internet. In figure 4 we have a first image. We display also the polar plane as it appears before the application of the algorithm (fig. 4-c) and after the elimination of the outliers (fig. 4-d).

In this example the vanishing point is marked with the white circle on the left side of the image in 4-b. It is clear from the polar plane that there are two vanishing points. One is more evident and is the one that has been estimated. The other one, which is on the extreme right of the image (outside the borders), is not very marked and then it does not influence the estimate. 
a)
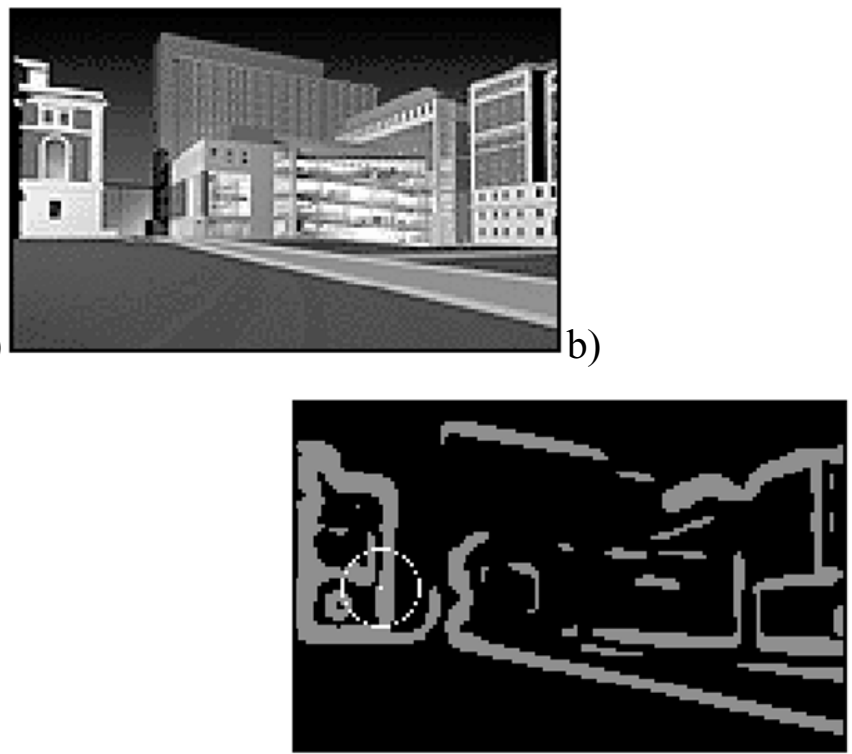

c)

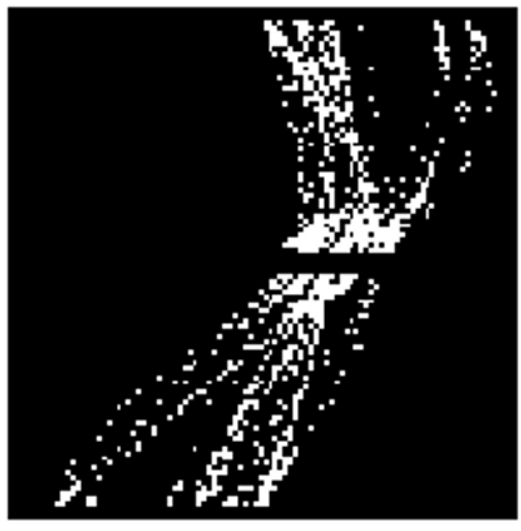

d)

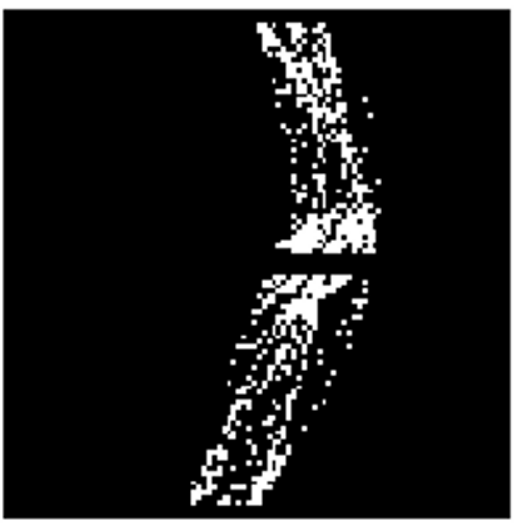

Fig. 4: In this artificial image we have a quite distinct vanishing point that is visible on image b). In the polar plane we can see in d) the sine curve that describes the vanishing point.

\section{Conclusions}

Through the estimation of the co-ordinates of the vanishing point many depth-related information's can be retrieved from a monocular image. The estimation of these coordinates can be done directly on the polar plane obtained with the Hough Transform. In fact in the polar plane the vanishing point is represented by a sine curve that can be 
detected with a statistical method. Filtering in the polar plane must not be done, hence the algorithm is independent from image structure and parameters.

\section{References}

1 H. Nakatani, S. Kimura, O. Saito, T. Kitahashi, Extraction of vanishing point and its application to scene analysis based on image sequence, Proceedings of $5^{\text {th }}$ International Conference on Pattern Recognition, 1980, pp. 370-372.

2 H.A. Sedgwick, Environment-Centered Representation of Spatial Layout: Available Visual Information from Texture and Perspective, in: J. Beck, B. Hope and A. Rosenfeld (Eds.), Human and Machine Vision, Accademic Press, New York (1983), pp. 425-428.

3 M.J. Magee and J.K. Aggarwal, Determining vanishing points from perspective images, Computer Vision, Graphics, and Image Processing, vol. 26, pp.256-267, 1984.

4 B. Brillault and O'Mahony, New method for vanishing points detection, CVGIP: Image Understanding, vol. 54, no.2, pp. 289-300, 1991.

5 P. Parodi and G. Piccioli, 3D shape reconstruction by using vanishing points, IEEE Transactions on Pattern Analysis and Machine Intelligence, vol. 18, no. 2, 1996.

6 P. V. Hough, Methods and means to recognize complex pattern. U.S. Patent 3,069,654, 1962.

7 D. Ballard and C. Brown, Computer Vision. Prentice-Hall, Englewood Cliffs, NJ, 1982. 